\title{
Stationary currents in long-range interacting magnetic systems
}

\section{Roberto Boccagna ${ }^{1}$ (i)}

Received: 19 November 2019 / Accepted: 20 July 2020 / Published online: 6 August 2020

(C) The Author(s) 2020

\begin{abstract}
We construct a solution for the $1 d$ integro-differential stationary equation derived from a finite-volume version of the mesoscopic model proposed in Giacomin and Lebowitz (J. Stat. Phys. 87(1), 37-61, 1997). This is the continuous limit of an Ising spin chain interacting at long range through Kac potentials, staying in contact at the two edges with reservoirs of fixed magnetizations. The stationary equation of the model is introduced here starting from the Lebowitz-Penrose free energy functional defined on the interval $\left[-\varepsilon^{-1}, \varepsilon^{-1}\right], \varepsilon>0$. Below the critical temperature, and for $\varepsilon$ small enough, we obtain a solution that is no longer monotone when opposite in sign, metastable boundary conditions are imposed. Moreover, the mesoscopic current flows along the magnetization gradient. This can be considered as an analytic proof of the existence of diffusion along the concentration gradient in one-component systems undergoing a phase transition, a phenomenon generally known as uphill diffusion. In our proof uniqueness is lacking, and we have clues that the stationary solution obtained is not unique, as suggested by numerical simulations.
\end{abstract}

Keywords Uphill diffusion · Kac potentials · Fick's law $\cdot$ Phase transitions

Mathematics Subject Classification (2010) $82 \mathrm{C} 26$

\section{Introduction}

The aim of this paper is to study Fick's law of transport in one-component systems undergoing a second order phase transition. In this context, it represents a step forward towards the establishment of a well posed theory for diffusion along the gradient (uphill diffusion) in the out-of-equilibrium setting [2-4]. Fick's law relates the flux $J$

Roberto Boccagna

robertoboccagna@gmail.com

1 Università dell'Aquila, Via Vetoio, Loc. Coppito, 67010 L'Aquila, Italia 
of a given substance to the gradient of its concentration $\rho$, which we suppose to be a differentiable function of the position in $[0, L]$ :

$$
J=-D \frac{\mathrm{d}}{\mathrm{d} x} \rho,
$$

at fixed boundary conditions $\rho(0)=\rho_{-}, \rho(L)=\rho_{+}$, with $\rho_{-}<\rho_{+}$without loss of generality. Here, $D>0$ is the diffusion coefficient. According to (1), the flux is always in the direction of decreasing gradient, i.e. from the region at higher concentration to the region at lower concentration. Thus, the solution of (1) connects monotonically $\rho_{-}$to $\rho_{+}$, as represented in a sketchy way in Fig. 1. Indeed, (1) should be modified when considering systems that consist of many components, since diffusion may be also affected by possible microscopic, chemical interactions among different substances. Evidences of unexpected behaviors have already been reported by Nernst [5], Onsager [6] and especially Darken [7-9], who performed a crucial experiment in the late 40's. His setup consisted of pairs of doped steels (Fe-Si with a different wt. \% of silicon, Fe-Si and Fe-Mn or Fe-Si and Fe-Mo) containing a small difference in the carbon concentration at the edges. The steels were welded together and eventually held in a furnace in order to let diffusion occur. Surprisingly, it was observed that carbon diffused in the direction of the gradient instead of against it in the mixtures with slightly differences in carbon concentration. This is shown in Fig. 2, which refers to the Fe-Si-Mn compound after two weeks the experiment started.

This behavior has a microscopic origin: in fact, silicon decreases the chemical affinity of carbon, while manganese increases it. This results in a driving force that acts in the opposite direction with respect to the concentration gradient and might beat the gradient, provided the difference in the carbon concentration at the edges is small. Such mechanism, which actually sustains uphill diffusion, works until dopants penetrate the weld; then, "standard" diffusion regime is restored. In formulae, this can be modeled replacing (1) with a vectorial relation:

$$
J_{i}=-c_{i} \sum_{j} L_{i j} \nabla \mu_{j}, \quad i=1, \ldots, n
$$

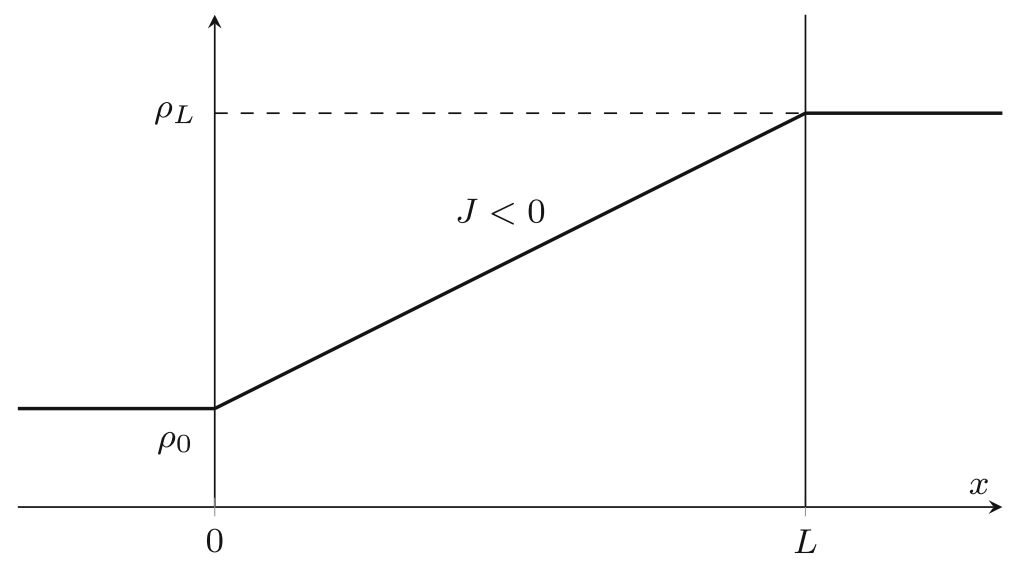

Fig. 1 Sketchy representation of the solution of (1) 


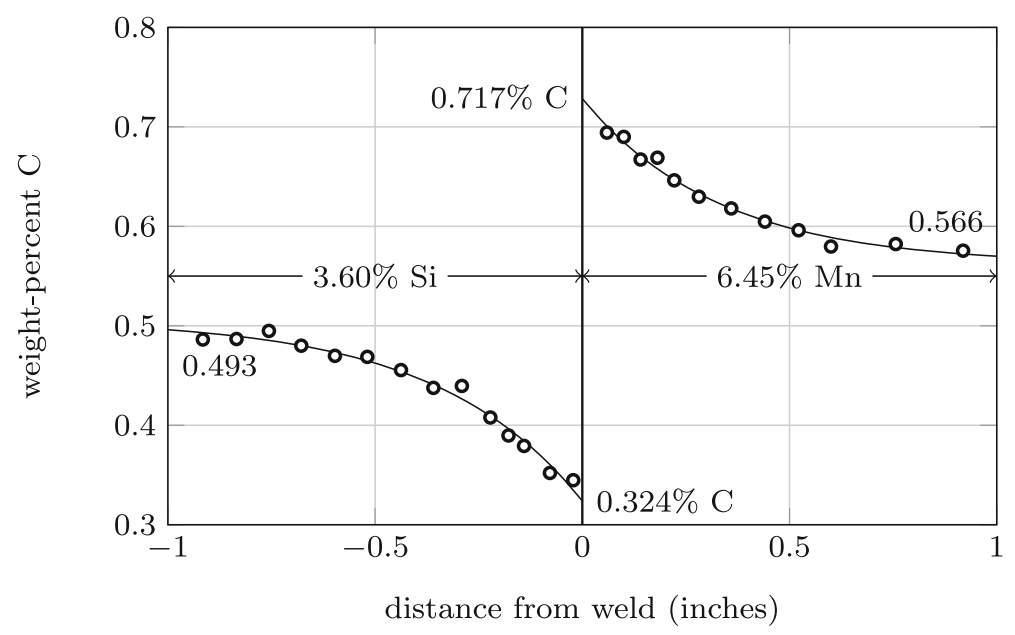

Fig. 2 Carbon concentration in the $\mathrm{Fe}-\mathrm{Si}-\mathrm{Mn}$ compound after 10 days at $1050{ }^{\circ} \mathrm{C}$ (figure extrapolated from [9])

where $n>2$ is the number of components of the system, $i$ refers to a given component, $c_{i}$ is the molar concentration of $i, L_{i j}$ represents the Fick diffusivity of $i$ given the presence of $j$, while the $\mu_{i}$ 's are chemical potentials. Equation (2) describes a system of $n-1$ linearly independent equations, because of the Gibbs-Duhem relation for chemical potentials $[10,11]$.

Numerical simulations suggest that uphill diffusion may also occur in onecomponent systems undergoing a phase separation. We mention in particular the dissipative version of the $1 d$ stochastic cellular automaton introduced by Colangeli et al. in [12] and the Ising spin chain with Kac potentials considered in [13], as these systems behave similarly. Stationary profiles have been obtained in the latter case by running a Kawasaki dynamics in which particles located at the edges may flip according to assigned rates. These are feasibly chosen in order to mimic interactions with reservoirs of infinite volume and opposite in sign magnetizations $\mu_{\text {left }}=-\mu$, $\mu_{\text {right }}=\mu, \mu \in(0,1)$. The evidence is that below the critical temperature, the current may flow along the magnetization gradient despite the fact that there is no external bias $^{1}$. This occurs provided $\mu$ belongs to an interval whose width depends on the temperature (the metastable region, see below). The corresponding steady profile is not monotone as one could expect (Fig. 3): an interface connects the minus to the plus phase, which regardless of the initial datum localizes after a transient in the neighborhood of one of the edges, randomly selected by dynamics. Although the current is positive, Fick's Law is actually violated only in the "small" region containing the interface, being the profile decreasing in the rest of the volume. Following [12], we call bumps profiles having this shape. Colangeli et al. obtained analogue numerical results for the $2 d$ nearest neighbors Ising model [14].

\footnotetext{
${ }^{1}$ The current is defined here as the signed variation of the total magnetization of the chain divided by the total time.
} 


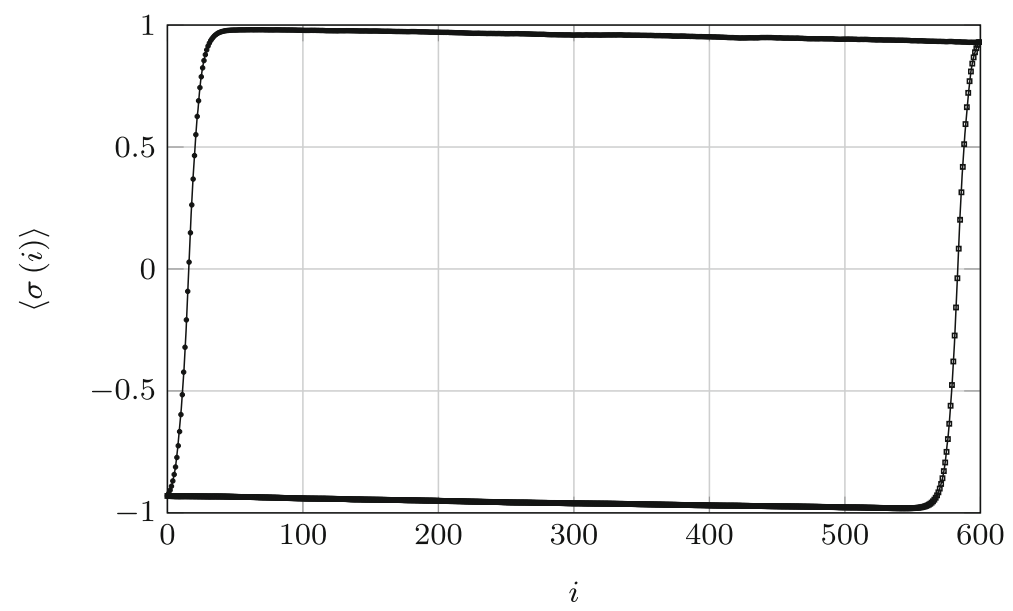

Fig. 3 Two steady magnetization profiles resulting from running Kawasaki dynamics for an Ising spin chain with $L=600$ sites for $\beta>1$. The boundaries are at constant values $\mu= \pm 0.93$

The microscopic mechanism underlying uphill diffusion in one-component systems has not a chemical origin. We speculate that the "force" that counteracts the gradient is provided in this case by the separation of phases; however, we evidence that such state is in fact metastable, in the sense that non-monotone profiles are local minima for the corresponding Gibbs free energy, but not global ones. We then expect that a large deviation eventually occurs and reverses the flux, although such inversion does not take place in the time considered for the mentioned simulations.

We establish here the existence of uphill diffusion in a model that is the continuous, mesoscopic limit of the Ising spin system with Kac potentials. The mesoscopic level represents an intermediate scale between the microscopic, discrete chain and the macroscopic model. We know that in the last case the phase diagram (i.e. the free energy density vs. magnetization diagram) has a global minimum for $\beta<1, \beta$ the inverse temperature in our units, while for $\beta>1$ the graph is flat in $\left[-m_{\beta}, m_{\beta}\right]$, $m_{\beta}$ the positive solution of the mean field equation $m=\tanh (\beta m)$. This indicates the occurrence of a phase transition. Any value in $\left(-m_{\beta}, m_{\beta}\right)$, the so called spinodal region, is then forbidden, so that any stationary profile containing values smaller of $-m_{\beta}$ and larger than $m_{\beta}$ must be discontinuous. At the mesoscopic level, the spinodal region is actually available and the discontinuity is replaced by a smooth interface, as proved by De Masi et al. for the free-boundary Stefan problem [15-18]. However, the discontinuity is recovered when the thermodynamic limit is performed; this consists in rescaling the space units to be proportional to the size of the system $L$ (macroscopic scaling), then letting $L$ diverge. In this sense, the mesoscopic model can be considered as a "smoothed" version of the macroscopic one [19].

The starting point of our analysis is the Lebowitz-Penrose (L-P) free energy functional, that is a non-local version of the scalar Ginzburg-Landau functional and that we postulate to describe the Physics of the system at the mesoscopic level. Under a gradient flow assumption, the magnetization evolves in time according to $\dot{m}=-\frac{\partial}{\partial x} I$, 
$I$ the local current which is assumed to be proportional to the functional derivative of the free energy (see Section 2.1). Fixing $\beta>1(\beta=1$ the critical inverse temperature in the mean-field limit), we study the stationary equation $\dot{m}=0$ in the interval $\left[-\varepsilon^{-1}, \varepsilon^{-1}\right], \varepsilon>0$, adopting Dirichlet, opposite in sign boundary conditions in analogy with the experimental scenario. Due to the form of the L-P functional, this turns out to be an integro-differential equation [1]. Using a functional version of Newton's method, we construct a solution restricting to the space of antisymmetric functions satisfying $m\left(\varepsilon^{-1}\right)=\mu, \mu$ in the region $\left(m^{*}(\beta), m_{\beta}\right)$. Here, $m^{*}(\beta)=\sqrt{1-1 / \beta}$ is the positive saddle point of the mean field free energy (Fig. 4). The profile that we obtain is monotone decreasing (then Fick's law holds locally there) excepting for a region of order $\varepsilon^{-\frac{1}{2}}$ in the neighborhood of the origin that contains the interface and moreover, the associated current flows uphill. The steps of our proof are sketchy described at the end of Section 3.1. It is worth stressing that our antisymmetric solution is supposedly unstable; in fact, numerical simulations suggest that bumps should be stable points for the corresponding gradient dynamics.

The same problem has already been studied in [22] although in the presence of an external, antisymmetric magnetic field that forces the solution to be antisymmetric as well. In that case it has been shown that, whatever the intensity of the external field, the flux is positive for any choice of metastable boundary conditions. De Masi et al. [19] considered the stationary Stefan problem in bounded domains, despite Neumann conditions have been adopted there. Apart from technicalities in the proof, the two approaches are quite different, since in the Neumann setting the magnetization profile naturally selects the boundary values imposed by the choice of current. However, there are solutions of the mesoscopic Neumann version of problem that converge to any solution of the Dirichlet problem as $\varepsilon \rightarrow 0$. We eventually point out that existence of uphill diffusion for the underlying microscopic model does not follow by our proof, and then a complete analytic description of such phenomenon is still missing.

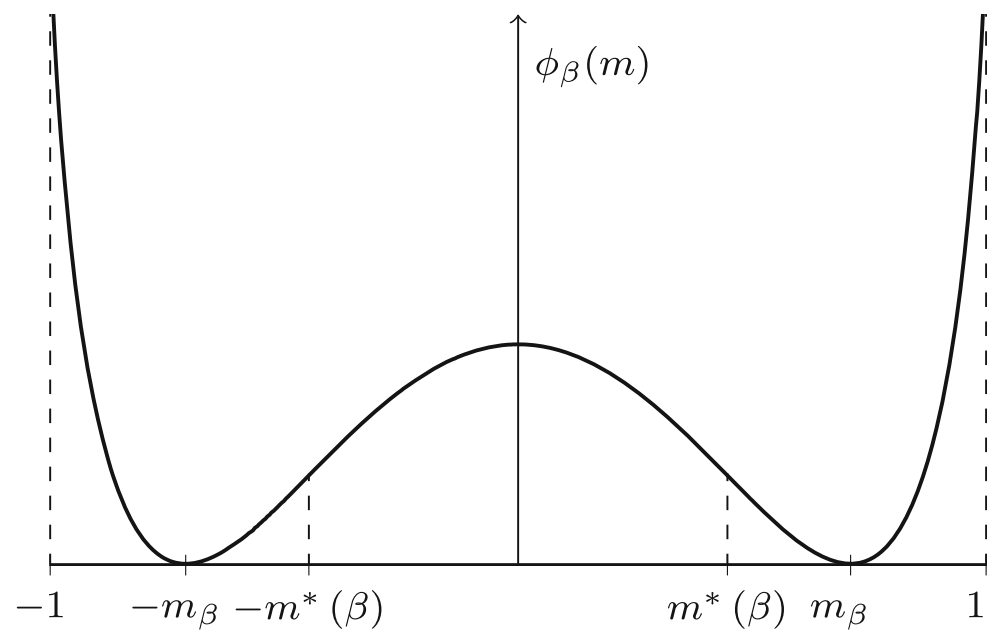

Fig. 4 The mean field free energy at $\beta>1$ 


\section{Background}

\subsection{The Lebowitz-Penrose Functional}

Indicate for notational convenience $\Lambda:=\left[-\varepsilon^{-1}, \varepsilon^{-1}\right]$ and $\Lambda^{c}:=\mathbb{R} \backslash\left[-\varepsilon^{-1}, \varepsilon^{-1}\right]$. Consider $m_{\Lambda} \in L^{\infty}(\Lambda,[-1,1]), m_{\Lambda^{c}} \in L^{\infty}\left(\Lambda^{c},[-1,1]\right), m_{\Lambda}$ being the magnetization density of the bulk and $m_{\Lambda^{c}}$ the magnetization of the reservoirs. Our starting point is the mesoscopic Lebowitz-Penrose free energy functional at zero external magnetic field, that is

$$
\begin{aligned}
& \mathcal{F}_{\beta}\left[m_{\Lambda} \mid m_{\Lambda^{c}}\right]=\mathcal{F}_{\beta}\left[m_{\Lambda}\right]+\frac{1}{2} \int_{\Lambda} \int_{\Lambda^{c}} \tilde{J}(x, y)\left[m_{\Lambda}(x)-m_{\Lambda^{c}}(y)\right]^{2} \mathrm{~d} x \mathrm{~d} y, \\
& \mathcal{F}_{\beta}\left[m_{\Lambda}\right]=\int_{\Lambda} \phi_{\beta}\left(m_{\Lambda}(x)\right) \mathrm{d} x+\frac{1}{4} \int_{\Lambda} \int_{\Lambda} \tilde{J}(x, y)\left[m_{\Lambda}(x)-m_{\Lambda}(y)\right]^{2} \mathrm{~d} x \mathrm{~d} y
\end{aligned}
$$

where

$$
\phi_{\beta}(m)=-\frac{1}{2} m^{2}-\frac{1}{\beta} S(m),
$$

and $S(m)$ is the standard binary entropy for an Ising spin system:

$$
S(m)=-\frac{1+m}{2} \log \left(\frac{1+m}{2}\right)-\frac{1-m}{2} \log \left(\frac{1-m}{2}\right) .
$$

$\tilde{J}$ is a probability kernel that depends on the distance between two points. The assumptions made on $\tilde{J}$ are precisely listed below:

- $\quad \tilde{J}$ is translational invariant and has a reflection symmetry, that is $\tilde{J}(x, y)=$ $\tilde{J}(0,|x-y|)$ for any $x, y \in \mathbb{R}$;

- $\quad \tilde{J}$ is twice differentiable, compactly supported: $\tilde{J} \in C_{K}^{2}([-1,1],[0,1])$;

- $\quad \tilde{J}$ is normalized, $\int_{\mathbb{R}} \tilde{J}(\cdot, y) \mathrm{d} y=1$;

- $\quad \tilde{J}(0, x)$ is strictly decreasing in $[0,1]$.

We treat expressions (3) and (4) as primitive quantities, by postulating them to define our model at a mesoscopic level. Indeed, it can be shown that this is precisely what one obtains taking the continuous limit of the underlying microscopic Ising chain with Kac potentials, and interacting at the boundaries with reservoirs of fixed magnetization densities, after a feasible scaling. We indicate reference [1] for details on this procedure, that is known as the Lebowitz-Penrose limit. We drop hereafter the suffix $\Lambda$, also indicating $\mathcal{F}_{\beta, \mu}[m] \equiv \mathcal{F}_{\beta}\left[m_{\Lambda} \mid m_{\Lambda^{c}}\right]$ for the sake of simplicity. According to the postulates of the axiomatic theory (see e.g. [19]), the magnetization density evolves in time according to a gradient dynamics

$$
\dot{m}(x, t)=-\frac{\partial I}{\partial x}(x, t), \quad t \geqslant 0
$$

where $I$ represents the current

$$
I(x, t):=-\chi_{\beta}(m(x, t)) \frac{\partial}{\partial x} \frac{\delta \mathcal{F}_{\beta, \mu}[m]}{\delta m(x, t)},
$$


in which $\chi_{\beta}(m)=\beta\left(1-m^{2}\right)$ is the mobility coefficient. Hence, the stationary problem $\dot{m}=0$ reads

$$
I=-\chi_{\beta}(m(x)) \frac{\partial}{\partial x} \frac{\delta \mathcal{F}_{\beta, \mu}[m]}{\delta m(x)},
$$

that is an integro-differential equation in the unknown function $m$ at constant $I$ and given boundary conditions. We write $I=j \varepsilon, j \in \mathbb{R}$ a constant, as we expect the current to be of order $\varepsilon$.

\subsection{Notation}

In this paragraph $m, h$ and $f$ are bounded functions in $\left[-\varepsilon^{-1}, \varepsilon^{-1}\right]$. We indicate

$$
\|f\|_{\varepsilon}:=\sup _{|x| \leqslant \varepsilon^{-1}}|f(x)|
$$

as the sup norm in that interval. In order to restrict our analysis on the interior of the bulk, we redefine the convolution kernel in the distributional sense:

$$
J(x, y) \stackrel{\mathcal{D}^{\prime}}{=} \tilde{J}(x, y) \mathbf{1}_{|y|<\varepsilon^{-1}}+b_{\varepsilon}(|x|)\left[\delta\left(\varepsilon^{-1}-y\right)+\delta\left(\varepsilon^{-1}+y\right)\right] \mathbf{1}_{|y| \geqslant \varepsilon^{-1}},
$$

where

$$
b_{\varepsilon}(x):=\int_{\varepsilon^{-1}}^{\varepsilon^{-1}+1} \tilde{J}(x, y) \mathrm{d} y .
$$

The convolution between $J$ and any bounded function is denoted in the usual way:

$$
(J * m)(x):=\int J(x, y) m(y) \mathrm{d} y
$$

and, unless otherwise indicated, integrals are intended to be computed on $\left[-\varepsilon^{-1}, \varepsilon^{-1}\right]$. The quantities

$$
p_{m, h}(x):=\beta\left(1-\tanh ^{2}\{\beta[(J * m)(x)+h(x)]\}\right)
$$

and

$$
p_{m, h}^{\prime}(x):=p_{m, h}(x) \tanh \{\beta[(J * m)(x)+h(x)]\} .
$$

play a crucial role in our analysis. We let $\mathrm{A}_{m, h}$ the linear operator with kernel $\mathrm{A}_{m, h}(x, y)=p_{m, h}(x) J(x, y)$ acting on bounded functions as follows

$$
\mathrm{A}_{m, h} f(x)=\int p_{m, h}(x) J(x, y) f(y) \mathrm{d} y .
$$

The action of the $n$-th power of $\mathrm{A}_{m, h}$ on $f$ is explicitly given by

$$
A_{m, h}^{n} f\left(x_{0}\right)=\int \prod_{i=1}^{n} p_{m, h}\left(x_{i-1}\right) J\left(x_{i-1}, x_{i}\right) f\left(x_{n}\right) \mathrm{d} x_{1} \ldots \mathrm{d} x_{n} .
$$




\subsection{Instantons}

We briefly recall a fundamental result obtained by De Masi et al. [15-18]. This regards the free boundary version of the problem. Let

$$
\mathcal{F}_{\beta}[m]=-\frac{1}{\beta} \int_{\mathbb{R}} \phi_{\beta}(m(x)) \mathrm{d} x+\frac{1}{4} \int_{\mathbb{R}} \int_{\mathbb{R}} \tilde{J}(x, y)[m(x)-m(y)]^{2} \mathrm{~d} x \mathrm{~d} y
$$

be the free energy functional on $\mathbb{R}$ defined for functions that belong to the space

$$
\mathcal{N}:=\left\{m \in L^{\infty}(\mathbb{R},[-1,1]) \mid \liminf _{x \rightarrow-\infty} m(x)<0, \limsup _{x \rightarrow+\infty} m(x)>0\right\} .
$$

In this case there are no external sources and the stationary equation reduces to

$$
\dot{m}(x, t)=-\frac{\delta \mathcal{F}_{\beta}[m]}{\delta m(x, t)}, \quad t \geqslant 0 .
$$

Let the instanton $\bar{m}$ the set of minimizers of $\mathcal{F}_{\beta}[m]$ in $\mathcal{N}$ solution of

$$
\bar{m}(x)=\tanh \{\beta[(\tilde{J} * \bar{m})(x)]\}, \quad x \in \mathbb{R}
$$

that satisfies $\lim _{x \rightarrow \pm \infty} m(x)= \pm m_{\beta}$, where $m_{\beta}$ is the positive solution of $m=$ $\tanh (\beta m)$. The following result is proved in [20], Ch. 8.

Theorem 2.1 For any $\beta>1$ the following holds:

(i) the variational problem $\delta \mathcal{F}_{\beta}[m]=0$ has a minimizer which is unique up to translations;

(ii) the mean field (20) has a solution in $\mathcal{N}$ which is unique in $\mathcal{N}$ up to translations;

(iii) for any $m \in \mathcal{N}$, there is $\xi \in \mathbb{R}$ so that $\lim _{t \rightarrow \infty}\left\|m(\cdot, t)-m_{\xi}\right\|_{\infty}=0$, where $\bar{m}_{\xi}(x):=\bar{m}(x-\xi) \cdot \bar{m} \in C^{\infty}(\mathbb{R},[-1,1])$ is a strictly increasing, antisymmetric function which converges exponentially fast to $\pm m_{\beta}$ as $x \rightarrow$ $\pm \infty$;

(iv) the function $\bar{m}$ defined in (iii) minimizes $\mathcal{F}_{\beta}[m]$ and it is therefore an istanton.

Hereafter, we call

$$
\bar{p}(x):=\beta\left[1-\bar{m}^{2}(x)\right], \quad \mathrm{A}_{\bar{m}}(x, y):=\bar{p}(x) \tilde{J}(x, y)
$$

for any $x$ and $y$ in $\mathbb{R}$. Similarly to (16), we indicate

$$
\mathrm{A}_{\bar{m}}^{n} f\left(x_{0}\right)=\int_{\mathbb{R}^{n}} \prod_{i=1}^{n} \bar{p}\left(x_{i-1}\right) \tilde{J}\left(x_{i-1}, x_{i}\right) f\left(x_{n}\right) \mathrm{d} x_{1} \ldots \mathrm{d} x_{n} .
$$

\section{Uphill Diffusion}

Our main result regards the existence of an antisymmetric solution $m$ of (9) with $I>0$, despite $m\left(-\varepsilon^{-1}\right)$ is in the minus phase and $m\left(\varepsilon^{-1}\right)$ in the plus phase. $m$ first 
decreases, then increases to jump into the plus region, then decreases again. Being the current positive, Fick's Law is violated only in the portion of the volume that contains the interface, which is of the order of $\varepsilon^{-\frac{1}{2}}$.

Theorem 3.1 At fixed $\beta>1$, for any $\mu \in\left(m^{*}(\beta), m_{\beta}\right)$ there are an antisymmetric, continuous function $m$ and $j>0$ that solve

$$
\left\{\begin{array}{l}
j \varepsilon=-\chi_{\beta}(m(x)) \frac{\partial}{\partial x} \frac{\delta \mathcal{F}_{\beta, \mu}[m]}{\delta m(x)}, \\
m\left(-\varepsilon^{-1}\right)=-\mu, \quad m\left(\varepsilon^{-1}\right)=\mu
\end{array}\right.
$$

if $\varepsilon$ is small enough.

\subsection{Outline of the Proof}

For our purposes, it is worth performing a change of variables:

$$
h(x):=\frac{\delta \mathcal{F}_{\beta, \mu}[m]}{\delta m(x)}
$$

where, explicitly

$$
\frac{\delta \mathcal{F}_{\beta, \mu}[m]}{\delta m(x)}=-\frac{1}{\beta} \operatorname{arctanh}(m(x))+\int J(x, y) m(y) \mathrm{d} y .
$$

In this position (9) becomes, after a straightforward integration

$$
h(x)=h\left(x_{0}\right)-j \varepsilon \int_{x_{0}}^{x} \frac{\mathrm{d} y}{\chi_{\beta}(m(y))} .
$$

Observe that $h(0)=0$ if $m$ is odd, so we can eventually formulate problem (23) as a system of coupled equations:

$$
\left\{\begin{array}{l}
m(x)=\tanh \{\beta[(J * m)(x)+h(x)]\} \\
h(x)=-j \varepsilon \int_{0}^{x} \frac{\mathrm{d} y}{\chi_{\beta}(m(y))}
\end{array}, \quad x \in\left[-\varepsilon^{-1}, \varepsilon^{-1}\right],\right.
$$

with $m\left(-\varepsilon^{-1}\right)=-\mu, m\left(\varepsilon^{-1}\right)=\mu$. Notice that the first equation in (27) is just (25) expressed in the new variables. We will often indicate

$$
\left[\mathrm{H}_{j}(m)\right](x):=-j \varepsilon \int_{0}^{x} \frac{\mathrm{d} y}{\chi_{\beta}(m(y))} \quad \forall x \in\left[-\varepsilon^{-1}, \varepsilon^{-1}\right] .
$$

The existence of a solution of (27) is proved through a functional version of Newton's method introduced in [23] and also used in [21, 22]: we start from a couple $\left(m_{0}, h_{0}\right)$ at given $\mu_{0}$ and $j$ ( $\mu_{0}$ and $j$ actually identify each other) and define a feasible map 
$\left(m_{n}, h_{n}\right) \mapsto\left(m_{n+1}, h_{n+1}\right)$ that converges uniformly to a couple $(m, h)$ that solves (27) and satisfies certain boundary conditions $m\left( \pm \varepsilon^{-1}\right)= \pm \mu$, with $\mu \neq \mu_{0}$ in general, while $j$ remains fixed in the iterations. Afterwards, we show that $j$ can be tuned in order to cover the whole metastable region, that is for any $\mu \in\left(m^{*}(\beta), m_{\beta}\right)$ there exists at least one $j>0$ such that $\lim _{n \rightarrow \infty} m_{n}(x)$ solves (27) with $m\left(\varepsilon^{-1}\right)=$ $\mu$. In this scheme, the choice of $m_{0}$ (and $h_{0}$ as a function of $m_{0}$ ) turns out to be crucial, as the starting profile has to be "almost" a fixed point.

The technical part of the paper is organized as follows: after having established the recursive method and chosen $m_{0}$, we perform in Section 4 some estimates that are needed in the course of the proof; in particular, we prove the invertibility of I $-\mathrm{A}_{m, h}$ for $\varepsilon$ small enough. In Section 5 we construct the sequence $\left(m_{n}, h_{n}\right)_{n=0}^{\infty}$ and prove its convergence to a certain solution of (27) with $j>0$. In Section 6 we deal with the invertibility issue mentioned above.

\subsection{Choice of the starting profile}

Proposition 3.2 The "macroscopic" problem at $\beta>1$

$$
\left\{\begin{array}{l}
j_{M}=-\left[1-\chi_{\beta}(M(r))\right] M^{\prime}(r), \quad r \in[0,1] \\
M(0)=\mu_{-}, \quad M(1)=\mu_{+}
\end{array}\right.
$$

with $0<\mu_{+}<\mu_{-}<1$, admits an unique solution $M(r)$ in $C^{\infty}([0,1])$. Such solution is decreasing in $[0,1]$.

Proof A straightforward integration gives

$$
r=-\frac{1}{j_{M}}\left[(\beta-1)\left(M(r)-\mu_{-}\right)-\frac{\beta}{3}\left(M^{3}(r)-\mu_{-}^{3}\right)\right],
$$

with $j_{M}$ fixed by the choice of $\mu_{-}$and $\mu_{+}$:

$$
j_{M}=(\beta-1)\left(\mu_{-}-\mu_{+}\right)-\frac{\beta}{3}\left(\mu_{-}^{3}-\mu_{+}^{3}\right) .
$$

As a function of $M, r$ is infinitely times differentiable and moreover, $r(M)$ is invertible since $M^{\prime}$ is negative. $M$ can be obtained as the unique real solution of the cubic equation (30).

We refer to (29) as the macroscopic equation because it comes from the variational problem that one obtains after performing the macroscopic limit (see [21, 22]). Notice that (29) can be formulated as a system of coupled equations as well

$$
\left\{\begin{array}{l}
M(r)=\tanh \{\beta[M(r)+H(r)]\} \\
H(r)=\tilde{H}-j_{M} \int_{0}^{r} \frac{\mathrm{d} r^{\prime}}{\chi_{\beta}\left(M\left(r^{\prime}\right)\right)}
\end{array}, \quad r \in[0,1]\right.
$$


with $\tilde{H}=\beta^{-1} \operatorname{arctanh}\left(\mu_{-}\right)-\mu_{-}$. We then define the starting profile as

$$
m_{0}(x):= \begin{cases}\bar{m}(x) & 0 \leqslant x \leqslant \varepsilon^{-\frac{1}{2}} \\ M_{\mu_{0}}\left(\frac{\varepsilon x-\sqrt{\varepsilon}}{1-\sqrt{\varepsilon}}\right)=: \hat{M}_{\mu_{0}}(x) & \varepsilon^{-\frac{1}{2}} \leqslant x \leqslant \varepsilon^{-1},\end{cases}
$$

with $m_{0}(x)=-m_{0}(-x)$ for $x<0$, where $M_{\mu_{0}}$ is the solution of (29) that satisfies $M_{\mu_{0}}(0)=\bar{m}\left(\varepsilon^{-\frac{1}{2}}\right)$ and $M_{\mu}(1)=\mu_{0}$.

Indeed, we speculate that if $\varepsilon^{-1}$ is large enough, the solution $m$ of (23) does not differ so much from the instanton in the neighborhood of the origin. Once reached the value $m_{0}\left(\varepsilon^{-\frac{1}{2}}\right)$, that for $\varepsilon^{-1}$ large is close to $m_{\beta}$, we suppose the profile to be monotone decreasing and "close" to the (rescaled) macroscopic solution. This will be clear a posteriori, as we will show that in fact the distance between $m_{0}$ and the stationary solution $m$ is of order $\varepsilon$ in the sup norm.

\subsection{Iterative Scheme}

The following results explicitly define the method.

Proposition 3.3 Let $m_{0}$ as in (33) satisfying $m_{0}\left(\varepsilon^{-1}\right)=\mu_{0}$, where $\mu_{0} \in$ $\left(m^{*}(\beta), m_{\beta}\right)$ and let $h_{0}=\mathrm{H}_{j}\left(m_{0}\right)$. If $\varepsilon$ is small enough, for any integer $n>1$, there is $m_{n} \in C\left(\left[-\varepsilon^{-1}, \varepsilon^{-1}\right],[-1,1]\right)$ that solves

$$
\left\{\begin{array}{l}
m_{n}(x)=\tanh \left\{\beta\left[\left(J * m_{n}\right)(x)+h_{n-1}(x)\right]\right\}, \quad x \in\left[-\varepsilon^{-1}, \varepsilon^{-1}\right] \\
h_{n-1}(x)=\left[\mathrm{H}_{j}\left(m_{n-1}\right)\right](x)
\end{array}\right.
$$

with $m_{n}\left(-\varepsilon^{-1}\right)=-\mu_{n}, m_{n}\left(\varepsilon^{-1}\right)=\mu_{n}, \mu_{n} \in\left(m^{*}(\beta), m_{\beta}\right)$. The sequence $\left(m_{n}, h_{n}\right)_{n=0}^{\infty}$ converges uniformly to a pair $(m, h)$, where $h=\mathrm{H}_{j}(m)$, which is a solution of problem (27) satisfying certain boundary conditions $m\left(-\varepsilon^{-1}\right)=-\mu$, $m\left(\varepsilon^{-1}\right)=\mu$, $\mu$ in the metastable region. Then, $m$ also solves (23) with the same boundary conditions.

Proposition 3.4 Under the same hypothesis of Proposition 3.3, for any value $\mu \in$ $\left(m^{*}(\beta), m_{\beta}\right)$ there is at least one $j>0$ such that the iterative scheme defined above converges to a solution of (23) with boundary conditions $m\left(-\varepsilon^{-1}\right)=-\mu$, $m\left(\varepsilon^{-1}\right)=\mu$ if $\varepsilon$ small enough.

In the iterations, $j=j\left(\mu_{0}\right)$ is a fixed parameter, whose value is specified by $m_{0}\left(\varepsilon^{-1}\right)=\mu_{0}\left(\right.$ and $\bar{m}\left(\varepsilon^{-\frac{1}{2}}\right)$ that depends on $\varepsilon$ only):

$$
j\left(\mu_{0}\right)=(\beta-1)\left(\bar{m}\left(\varepsilon^{-\frac{1}{2}}\right)-\mu_{0}\right)-\frac{\beta}{3}\left(\bar{m}^{3}\left(\varepsilon^{-\frac{1}{2}}\right)-\mu_{0}^{3}\right) .
$$

At fixed $\varepsilon, j$ is then a non increasing function of $\mu_{0}$ having its unique zero in $\bar{m}\left(\varepsilon^{-\frac{1}{2}}\right)$. Moreover, in the metastable interval $j$ is bounded from above by $j_{\max }:=j\left(m^{*}(\beta)\right)$. 
In order to have positive current, we take in the sequel $\varepsilon$ so small that $m_{0}\left(\varepsilon^{-\frac{1}{2}}\right)>\mu_{0}$. This is always possible by Theorem 2.1.

\section{Some Properties of $A_{m, h}$}

\subsection{A Preliminary Result}

We recall here a result proved in [24] and that can be even found in [20]. Define the scalar product on $\mathbb{R}$

$$
\langle f\rangle_{\infty}:=\int_{\mathbb{R}} f(x) \frac{\mathrm{d} x}{\bar{p}(x)}
$$

and denote $\tilde{m}^{\prime}:=\bar{m}^{\prime} / \sqrt{\left\langle\left(\bar{m}^{\prime}\right)^{2}\right\rangle_{\infty}}$, where $\bar{m}^{\prime}$ is the first derivative of $\bar{m}$. We have the following:

Proposition 4.1 There are positive constants $a$ and $c$ such that for any $f \in L^{\infty}(\mathbb{R})$ and any integer $n$ :

$$
\left|\int_{\mathbb{R}^{n}} \mathrm{~A}_{\bar{m}}^{n}(x, y) \tilde{f}(y) \mathrm{d} y\right| \leqslant c\|\tilde{f}\|_{\infty} \mathrm{e}^{-a n}, \quad \tilde{f}:=f-\left\langle f \tilde{m}^{\prime}\right\rangle_{\infty} \tilde{m}^{\prime} .
$$

There is a very straight consequence of this result, which is however essential for our purposes.

Corollary 4.2 For any bounded, antisymmetric function $\psi$ on $\mathbb{R}$ and any integer $n:$

$$
\left|\int_{\mathbb{R}^{n}} \mathrm{~A}_{\bar{m}}^{n}(x, y) \psi(y) \mathrm{d} y\right| \leqslant c \mathrm{e}^{-a n}\|\psi\|_{\infty} .
$$

Proof Since $\tilde{m}^{\prime}$ is symmetric, $\left\langle\psi \tilde{m}^{\prime}\right\rangle_{\infty}=0$ and then $\tilde{\psi} \equiv \psi$.

\subsection{Estimates on the Starting Profile}

Proposition 4.3 Let $m_{0}$ as in (33) with $m\left(\varepsilon^{-1}\right)=\mu_{0}$ and $h_{0}=\mathrm{H}_{j}\left(m_{0}\right)$. The following estimates hold:

$$
\begin{aligned}
& \left|p_{m_{0}, h_{0}}(x)-\bar{p}(x)\right| \leqslant \frac{4 \beta^{2} j}{\chi_{\beta}\left(m_{\beta}\right)} \sqrt{\varepsilon}, \quad|x| \leqslant \varepsilon^{-\frac{1}{2}} ; \\
& \left|p_{m_{0}, h_{0}}(x)-\chi_{\beta}\left(m_{0}(x)\right)\right| \leqslant \frac{4 \beta^{2} j}{1-\chi_{\beta}\left(\mu_{0}\right)} \frac{\varepsilon}{1-\sqrt{\varepsilon}}, \quad \varepsilon^{-\frac{1}{2}} \leqslant|x| \leqslant \varepsilon^{-1} .
\end{aligned}
$$

Proof We know that for any real numbers $x_{1}$ and $x_{2}$,

$$
\left|\tanh ^{2}\left(x_{2}\right)-\tanh ^{2}\left(x_{1}\right)\right| \leqslant 4 \tanh \left|x_{2}-x_{1}\right| .
$$


Since $m_{0} \equiv \bar{m}$ in $\left[-\varepsilon^{-\frac{1}{2}}, \varepsilon^{-\frac{1}{2}}\right]$, using (41) we get

$$
\left|p_{m_{0}, h_{0}}(x)-\bar{p}(x)\right| \leqslant 4 \beta \tanh \left|\beta\left[j \varepsilon \int_{0}^{x} \frac{\mathrm{d} y}{\chi_{\beta}\left(m_{0}(y)\right)}\right]\right| \leqslant \frac{4 \beta^{2} j}{\chi_{\beta}\left(m_{\beta}\right)} \sqrt{\varepsilon},
$$

because $\left\|m_{0}\right\|_{\varepsilon}<m_{\beta}$ and then $\inf _{|x| \leq \varepsilon^{-1}}\left|\chi_{\beta}\left(m_{0}(x)\right)\right|_{\varepsilon}>\chi_{\beta}\left(m_{\beta}\right)$. For any $\varepsilon^{-\frac{1}{2}} \leqslant$ $|x| \leqslant \varepsilon^{-1}$,

$$
\begin{aligned}
\left|p_{m_{0}, h_{0}}(x)-\chi_{\beta}\left(m_{0}(x)\right)\right| & =\beta\left|\tanh ^{2}\left\{\beta\left[\left(J * \hat{M}_{\mu_{0}}\right)(x)+h_{0}(x)\right]\right\}-\hat{M}_{\mu_{0}}^{2}(x)\right| \\
& \leqslant 4 \beta^{2}\left|\left(J * \hat{M}_{\mu_{0}}\right)(x)-\hat{M}_{\mu_{0}}(x)\right| \\
& \leqslant 4 \beta^{2} \max _{y:|y-x| \leq 1}\left|\hat{M}_{\mu_{0}}(y)-\hat{M}_{\mu_{0}}(x)\right| \\
& \leqslant 4 \beta^{2}\left\|\hat{M}_{\mu_{0}}^{\prime}\right\|_{\varepsilon}=4 \beta^{2}\left\|M_{\mu_{0}}\right\|_{\varepsilon} \frac{\varepsilon}{1-\sqrt{\varepsilon}}
\end{aligned}
$$

where in the last line we used Lagrange's theorem and (33). Since $M_{\mu_{0}}$ is non increasing, we deduce from (29) that $\left\|M_{\mu_{0}}\right\|_{\varepsilon} \leq j /\left(1-\chi_{\beta}\left(\mu_{0}\right)\right)$.

Hereafter, we tacitly suppose for technical reasons that $\varepsilon$ is so small that

$$
\begin{aligned}
& \mathrm{e}^{-a}\left(1+\frac{4 \beta^{2} j}{\chi_{\beta}^{2}\left(m_{\beta}\right)} \sqrt{\varepsilon}\right)<1 \\
& \left\|\chi_{\beta}\left(m_{0}\right)\right\|_{\varepsilon}+\frac{4 \beta^{2} j}{\chi_{\beta}^{2}\left(m_{\beta}\right)} \sqrt{\varepsilon}<1 \\
& \left\|\chi_{\beta}\left(m_{0}\right)\right\|_{\varepsilon}+\frac{4 \beta^{2} j}{1-\chi_{\beta}\left(\mu_{0}\right)} \frac{\varepsilon}{1-\sqrt{\varepsilon}}<1 .
\end{aligned}
$$

\subsection{Invertibility of the Main Operator}

At fixed $\delta>0$, let

$$
\Sigma_{\delta}:=\left\{p \in L^{\infty}\left(\left[-\varepsilon^{-1}, \varepsilon^{-1}\right]\right) \mid\left\|p-p_{m_{0}, h_{0}}\right\|_{\varepsilon}<\delta\right\} .
$$

For any $p \in \Sigma_{\delta}$, we call A the linear operator with kernel $\mathrm{A}(x, y)=p(x) J(x, y)$.

Proposition 4.4 Let $p \in \Sigma_{\delta}$. For $\delta$ small enough, there exists a constant $\theta=\theta(\delta)<$ 1 such that for any bounded, antisymmetric function $\psi$ on $\left[-\varepsilon^{-1}, \varepsilon^{-1}\right]$ and any integer $n$ :

$$
\left|\int_{\substack{\left|x_{i}\right| \leqslant \varepsilon^{-\frac{1}{2}} \\ i=1, \ldots, n}} \prod_{i=1}^{n} \mathrm{~A}\left(x_{i-1}, x_{i}\right) \psi\left(x_{n}\right) \mathrm{d} x_{1} \ldots \mathrm{d} x_{n}\right| \leqslant c \mathrm{e}^{-a n} \theta^{n} \sup _{\left|x_{0}\right| \leqslant \varepsilon^{-\frac{1}{2}}}\left|\psi\left(x_{0}\right)\right| .
$$


Proof We have, at fixed $x_{0} \in\left[-\varepsilon^{-\frac{1}{2}}, \varepsilon^{-\frac{1}{2}}\right]$ :

$$
\begin{aligned}
& \int\left|\prod_{\substack{\left|x_{i}\right| \leqslant \varepsilon^{-\frac{1}{2}} \\
i=1, \ldots, n}}\right| \mathrm{A}\left(x_{i-1}, x_{i}\right)-\prod_{i=1}^{n} \mathrm{~A}_{\bar{m}}\left(x_{i-1}, x_{i}\right) \mid \psi\left(x_{n}\right) \mathrm{d} x_{1} \ldots \mathrm{d} x_{n} \\
\leqslant & \int_{\substack{\left|x_{i}\right| \leqslant \varepsilon^{-\frac{1}{2}} \\
i=1, \ldots, n}}\left|\prod_{i=1}^{n} p\left(x_{i-1}\right) J\left(x_{i-1}, x_{i}\right)-\prod_{i=1}^{n} \bar{p}\left(x_{i-1}\right) J\left(x_{i-1}, x_{i}\right)\right| \psi\left(x_{n}\right) \mathrm{d} x_{1} \ldots \mathrm{d} x_{n} \\
\leqslant & \int_{\substack{\left|x_{i}\right| \leqslant \varepsilon^{-\frac{1}{2}} \\
i=1, \ldots, n}}\left|\prod_{i=1}^{n} \frac{p_{m, h}\left(x_{i-1}\right)}{\bar{p}\left(x_{i-1}\right)}-1\right| \prod_{i=1}^{n} \mathrm{~A}_{\bar{m}}\left(x_{i-1}, x_{i}\right) \psi\left(x_{n}\right) \mathrm{d} x_{1} \ldots \mathrm{d} x_{n} .
\end{aligned}
$$

Write

$$
\frac{p_{m, h}(x)}{\bar{p}(x)}=1++\frac{\left|p_{m_{0}, h_{0}}(x)-\bar{p}(x)\right|}{\bar{p}(x)}+\frac{\left|p_{m, h}(x)-p_{m_{0}, h_{0}}(x)\right|}{\bar{p}(x)} .
$$

By (39),

$$
\sup _{|x| \leqslant \varepsilon^{-\frac{1}{2}}} \frac{p_{m, h}(x)}{\bar{p}(x)} \leqslant 1+\frac{4 \beta^{2} j}{\chi_{\beta}\left(m_{\beta}\right)} \sqrt{\varepsilon}+\frac{1}{\chi_{\beta}\left(m_{\beta}\right)} \delta=: \theta,
$$

which is less than 1 for $\delta$ small because of (44). Therefore,

$$
\begin{aligned}
& \int \prod_{\substack{\left|x_{i}\right| \leqslant \varepsilon^{-\frac{1}{2}} \\
i=1, \ldots, n}}\left|\prod_{i=1}^{n} \mathrm{~A}\left(x_{i-1}, x_{i}\right)-\prod_{i=1}^{n} \mathrm{~A}_{\bar{m}}\left(x_{i-1}, x_{i}\right)\right| \psi\left(x_{n}\right) \mathrm{d} x_{1} \ldots \mathrm{d} x_{n} \\
\leqslant & \left(\theta^{n}-1\right) \prod_{\substack{\left|x_{i}\right| \leqslant \varepsilon^{-\frac{1}{2}} \\
i=1, \ldots, n}}^{n} \mathrm{~A}_{\bar{m}}\left(x_{i-1}, x_{i}\right) \psi\left(x_{n}\right) \mathrm{d} x_{1} \ldots \mathrm{d} x_{n} \\
\leqslant & c \mathrm{e}^{-a n}\left(\theta^{n}-1\right) \sup _{\left|x_{0}\right| \leqslant \varepsilon^{-\frac{1}{2}}}\left|\psi\left(x_{0}\right)\right|,
\end{aligned}
$$

where we used (38). Since $\left|\mathrm{A}^{n} \psi\right| \leqslant\left|\mathrm{A}_{\bar{m}}^{n} \psi\right|+\left|\left(\mathrm{A}^{n}-\mathrm{A}_{\bar{m}}^{n}\right) \psi\right|$, combining (52) with (38) we get the result.

Proposition 4.5 Fix $n_{\varepsilon}:=\left\lfloor\frac{\varepsilon^{-\frac{1}{2}}}{4}\right\rfloor$ and let $p \in \Sigma_{\delta}$. For $\delta$ small enough, there is $\gamma=\gamma(\delta)<1$ such that for any bounded, antisymmetric function $\psi$ on $\left[-\varepsilon^{-1}, \varepsilon^{-1}\right]$ and any $n \leqslant n_{\varepsilon}$ :

$$
\left|\int \prod_{i=1}^{n} \mathrm{~A}\left(x_{i-1}, x_{i}\right) \psi\left(x_{n}\right) \mathrm{d} x_{1} \ldots \mathrm{d} x_{n}\right| \leqslant C \gamma^{n}\|\psi\|_{\varepsilon}
$$

for any $x_{0} \in\left[-\varepsilon^{-1}, \varepsilon^{-1}\right], C>0$ a constant. 
Proof We distinguish two cases. If $\left|x_{0}\right| \leqslant \frac{3}{4} \varepsilon^{-\frac{1}{2}}$, then $\left|x_{i}\right| \leqslant \varepsilon^{-\frac{1}{2}}$ for any $i=$ $1, \ldots, n$ because $J$ has range 1, thus estimate (48) applies with $C=c$ and $\gamma=\mathrm{e}^{-a} \theta$.

Conversely, if $\frac{3}{4} \varepsilon^{-\frac{1}{2}} \leqslant\left|x_{0}\right| \leqslant \varepsilon^{-1}$, then $\left|x_{i}\right| \geqslant \frac{\varepsilon^{-\frac{1}{2}}}{2}$ for any $i=1, \ldots, n$ and we directly bound $p$ starting from the trivial inequality

$$
\left|p\left(x_{0}\right)\right| \leqslant \chi_{\beta}\left(m_{0}\left(x_{0}\right)\right)+\left|p_{m_{0}, h_{0}}\left(x_{0}\right)-\chi_{\beta}\left(m_{0}\left(x_{0}\right)\right)\right|+\mid p_{m_{0}, h_{0}}\left(x_{0}\right)-p\left(x_{0}\right)(54)
$$

In (54), the sum of the first two terms is always less than 1 because of (39) and (40) and by hypothesis (45) and (46). Therefore, if $\delta$ is small enough there exists a constant $\theta^{\prime}=\theta^{\prime}(\delta)<1$ that bounds $p\left(x_{i}\right)$ for any $\frac{3}{4} \varepsilon^{-\frac{1}{2}} \leqslant\left|x_{0}\right| \leqslant \varepsilon^{-1}$. Define $\gamma$ as the maximum between $\mathrm{e}^{-a} \theta$ and $\theta^{\prime}$ and choose $C=\max \{c, 1\}$ to obtain (53).

Proposition 4.5 induces the following

Proposition 4.6 let $p \in \Sigma_{\delta}$. For any bounded, antisymmetric function $\psi$ on $\left[-\varepsilon^{-1}, \varepsilon^{-1}\right]$ and for any $x_{0} \in\left[-\varepsilon^{-1}, \varepsilon^{-1}\right]$ :

$$
\left|\sum_{n=0}^{\infty} \int \prod_{i=0}^{n} \mathrm{~A}\left(x_{i-1}, x_{i}\right) \psi\left(x_{n}\right) \mathrm{d} x_{1} \ldots \mathrm{d} x_{n}\right| \leqslant \frac{C}{1-c_{\varepsilon} \gamma}\|\psi\|_{\varepsilon}
$$

if $\delta$ and $\varepsilon$ are small enough, where $c_{\varepsilon}$ is a constant.

Proof Write $n=k_{n} n_{\varepsilon}+r_{n}$, with $r_{n}<n_{\varepsilon}$ so that

$$
\begin{aligned}
\left\|\mathrm{A}^{n} \psi\right\|_{\varepsilon} \leqslant & \sup _{\left|x_{0}\right| \leqslant \varepsilon^{-1}} \mid \int \prod_{i_{1}=1}^{n_{\varepsilon}} \mathrm{A}\left(x_{i_{1}-1}, x_{i_{1}}\right) \mathrm{d} x_{1} \ldots \mathrm{d} x_{n_{\varepsilon}} \\
& \times \int \prod_{i_{2}=n_{\varepsilon}+1}^{2 n_{\varepsilon}} \mathrm{A}\left(x_{i_{2}-1}, x_{i_{2}}\right) \mathrm{d} x_{n_{\varepsilon}+1} \ldots \mathrm{d} x_{2 n_{\varepsilon}} \\
& \vdots \\
& \times \int \prod_{i_{k}=(k-1) n_{\varepsilon}+1}^{k n_{\varepsilon}} \mathrm{A}\left(x_{i_{k}-1}, x_{i_{k}}\right) \mathrm{d} x_{(k-1) n_{\varepsilon}+1} \ldots \mathrm{d} x_{k n_{\varepsilon}} \\
& \times \int \prod_{j=k n_{\varepsilon}+1}^{n} \mathrm{~A}\left(x_{j-1}, x_{j}\right) \psi\left(x_{n}\right) \mathrm{d} x_{k n_{\varepsilon}+1} \ldots \mathrm{d} x_{n} \mid .
\end{aligned}
$$

Call

$$
\Psi^{(k)}\left(x_{k n_{\varepsilon}}\right):=\int \prod_{j=k n_{\varepsilon}+1}^{n} \mathrm{~A}\left(x_{j-1}, x_{j}\right) \psi\left(x_{n}\right) \mathrm{d} x_{k n_{\varepsilon}+1} \ldots \mathrm{d} x_{n}
$$

and notice that $\Psi^{(k)}$ is antisymmetric and satisfies $\left\|\Psi^{(k)}\right\|_{\varepsilon} \leqslant C \gamma^{r_{n}}\|\psi\|_{\varepsilon}$ by virtue of (53). Thus, for any $i<k$ the function defined by

$$
\Psi^{(i)}\left(x_{i n_{\varepsilon}}\right):=\int \prod_{j=i n_{\varepsilon}+1}^{(i+1) n_{\varepsilon}} \mathrm{A}\left(x_{j-1}, x_{j}\right) \Psi^{(i+1)}\left(x_{n}\right) \mathrm{d} x_{i n_{\varepsilon}+1} \ldots \mathrm{d} x_{(i+1) n_{\varepsilon}}
$$


is antisymmetric and therefore by iterating (53)

$$
\begin{aligned}
\left\|\mathrm{A}^{n} \psi\right\|_{\varepsilon} & \leqslant \sup _{\left|x_{0}\right| \leq \varepsilon^{-1}} \mid \int \prod_{i_{1}=1}^{n_{\varepsilon}} \mathrm{A}\left(x_{i_{1}-1}, x_{i_{1}}\right) \Psi^{(1)}\left(x_{n_{\varepsilon}}\right) \mathrm{d} x_{1} \ldots \mathrm{d} x_{n_{\varepsilon}} \\
& \leqslant\left(C^{\frac{1}{n_{\varepsilon}}} \gamma\right)^{n_{\varepsilon}}\left\|\Psi^{(1)}\right\|_{\varepsilon} \leqslant\left(C^{\frac{1}{n_{\varepsilon}}} \gamma\right)^{2 n_{\varepsilon}}\left\|\Psi^{(2)}\right\|_{\varepsilon} \leqslant \ldots \leqslant\left(C^{\frac{1}{n_{\varepsilon}}} \gamma\right)^{k n_{\varepsilon}}\left\|\Psi^{(k)}\right\|_{\varepsilon} \\
& \leqslant C\left(C^{\frac{1}{n_{\varepsilon}}} \gamma\right)^{n}\|\psi\|_{\varepsilon} .
\end{aligned}
$$

Call $c_{\varepsilon}:=C^{\frac{1}{n_{\varepsilon}}}$ and let $\varepsilon$ so small that $c_{\varepsilon} \gamma<1$. Summing on $n$ we get (55).

The previous bound is crucial as it induces the existence of the inverse of I - A. The result that follows will be repeatedly used hereinafter.

Lemma 4.7 There is $\delta^{*}>0$ such that for any $\delta<\delta^{*}$ the following holds. Let $p \in \Sigma_{\delta}$. Then,

(i)

$$
(\mathrm{I}-\mathrm{A})^{-1}:=\sum_{k=0}^{\infty} \mathrm{A}^{k}, \quad\left\|(\mathrm{I}-\mathrm{A})^{-1}\right\|_{\varepsilon} \leqslant \frac{C}{1-c_{\varepsilon} \gamma} ;
$$

(ii) for any bounded, antisymmetric function $F$ on $\left[-\varepsilon^{-1}, \varepsilon^{-1}\right]$ the equation

$$
\varphi(x)-p(x)(J * \varphi)(x)=F(x)
$$

can be solved in the unknown function $\varphi$. Furthermore,

$$
\|\varphi\|_{\varepsilon} \leqslant \frac{C}{1-c_{\varepsilon} \gamma}\|F\|_{\varepsilon} .
$$

Proof Is a straightforward consequence of Proposition 4.6.

\subsection{Useful Lemmas}

Lemma 4.8 Let $^{(1)}, m^{(2)} \in L^{\infty}\left(\left[-\varepsilon^{-1}, \varepsilon^{-1}\right],[-1,1]\right)$. Then

$$
\left\|\mathrm{H}_{j}\left(m^{(2)}\right)-\mathrm{H}_{j}\left(m^{(1)}\right)\right\|_{\varepsilon} \leqslant c^{\prime} j\left\|m^{(2)}-m^{(1)}\right\|_{\varepsilon}, \quad c^{\prime}=\frac{2 \beta}{\chi_{\beta}^{2}\left(m_{\beta}\right)} .
$$

Proof We have, for any $x \in\left[-\varepsilon^{-1}, \varepsilon^{-1}\right]$ :

$$
\begin{aligned}
\left|\left[\mathrm{H}_{j}\left(m^{(2)}\right)\right](x)-\left[\mathrm{H}_{j}\left(m^{(1)}\right)\right](x)\right| & \leqslant \beta j \varepsilon \int_{0}^{x} \frac{\left|\left(m^{(2)}\right)^{2}(y)-\left(m^{(1)}\right)^{2}(y)\right|}{\chi_{\beta}\left(m^{(1)}(y)\right) \chi_{\beta}\left(m^{(2)}(y)\right)} \mathrm{d} y \\
& \leqslant \frac{2 \beta j}{\chi_{\beta}^{2}\left(m_{\beta}\right)}\left\|m^{(1)}-m^{(2)}\right\|_{\varepsilon} .
\end{aligned}
$$


Lemma 4.9 Let $m \in L^{\infty}\left(\left[-\varepsilon^{-1}, \varepsilon^{-1}\right]\right)$ and $h=\mathrm{H}_{j}(m)$ and suppose that $\left\|m-m_{0}\right\|_{\varepsilon} \leqslant \delta$. Then,

$$
\left\|p_{m, h}-p_{m_{0}, h_{0}}\right\| \leqslant c^{\prime \prime} \delta, \quad c^{\prime \prime}=4 \beta^{2}\left(1+j_{\max } c^{\prime}\right) .
$$

Proof Using (41), we estimate for any $x \in\left[-\varepsilon^{-1}, \varepsilon^{-1}\right]$ :

$$
\begin{aligned}
\left|p_{m, h}(x)-p_{m_{0}, h_{0}}(x)\right| & \leqslant 4 \beta \tanh \left|\beta\left[\left(J *\left(m-m_{0}\right)\right)(x)-\left(h(x)-h_{0}(x)\right)\right]\right| \\
& \leqslant 4 \beta^{2}\left(\left|m(x)-m_{0}(x)\right|+\left|h(x)-h_{0}(x)\right|\right) \\
& \leqslant 4 \beta^{2}\left(1+c^{\prime} j\right) \delta^{\prime}
\end{aligned}
$$

where we used (64).

\section{Newton's Method}

\subsection{Small Perturbations to $m_{0}$}

In this section we construct $m_{1}$ (the solution of (34) with $n=1$ ) as a series

$$
m_{1}=m_{0}+\sum_{k=0}^{\infty} \varphi_{k} ; \quad \phi_{k}:=\sum_{l=1}^{k} \varphi_{l}, \quad k \geqslant 1
$$

in which each correction $\varphi_{n}$ depends on the previous ones and $\varphi_{0} \equiv 0$ by definition. The well posedness of the method comes from the fact that for any $k$, $\left\|p_{m_{0}+\phi_{k}, h_{0}}-p_{m_{0}, h_{0}}\right\|_{\varepsilon}$ can be controlled in such a way that Lemma 4.7 applies.

Proposition 5.1 For any $\varepsilon$ small enough, there exists an antisymmetric function $\varphi_{1} \in$ $L^{\infty}\left(\left[-\varepsilon^{-1}, \varepsilon^{-1}\right]\right)$ that solves

$$
\left\{\begin{array}{l}
\varphi_{1}(x)-p_{m_{0}, h_{0}}(x)\left(J * \varphi_{1}\right)(x)=\tanh \left\{\beta\left[\left(J * m_{0}\right)(x)+h_{0}(x)\right]\right\}-m_{0}(x) \\
\varphi_{1}\left(-\varepsilon^{-1}\right)=-t_{1}, \quad \varphi_{1}\left(\varepsilon^{-1}\right)=t_{1}
\end{array}\right.
$$

for some $t_{1} \in \mathbb{R}$. Moreover, there is $c_{0}>0$ such that $\left\|\varphi_{1}\right\|_{\varepsilon} \leqslant c_{0} \varepsilon$.

Proof Call

$$
S_{0}(x):=\tanh \left\{\beta\left[\left(J * m_{0}\right)(x)+h_{0}(x)\right]\right\}-m_{0}(x) .
$$

We restrict w.l.o.g. to the positive semiline. We have, for any $0 \leqslant x \leqslant \varepsilon^{-\frac{1}{2}}-1$ :

$$
\begin{aligned}
\left|S_{0}(x)\right| & =\left|\tanh \left\{\beta\left[(J * \bar{m})(x)+h_{0}(x)\right]\right\}-\bar{m}(x)\right| \\
& \leqslant \beta j \varepsilon \int_{0}^{x} \frac{\mathrm{d} y}{\chi_{\beta}(\bar{m}(y))}=O(\sqrt{\varepsilon}) .
\end{aligned}
$$


In the interval $\varepsilon^{-\frac{1}{2}}+1 \leqslant x \leqslant \varepsilon^{-1}$, similarly to estimate (43) we get:

$$
\left|S_{0}(x)\right|=\left|\tanh \left\{\beta\left[\left(J * \hat{M}_{\mu_{0}}\right)(x)+h_{0}(x)\right]\right\}-\hat{M}_{\mu_{0}}(x)\right|=O(\varepsilon) .
$$

The remaining case is when $\varepsilon^{-\frac{1}{2}}-1 \leqslant x \leqslant \varepsilon^{-\frac{1}{2}}+1$. We consider at first the sub-case $\varepsilon^{-\frac{1}{2}}-1 \leqslant x \leqslant \varepsilon^{-\frac{1}{2}}$. We have

$$
\begin{aligned}
\left|S_{0}(x)\right|= & \tanh \left\{\beta\left[\int_{x-1}^{\varepsilon^{-\frac{1}{2}}} J(x, y) \bar{m}(y) \mathrm{d} y+\int_{\varepsilon^{-\frac{1}{2}}}^{x+1} J(x, y) \hat{M}_{\mu_{0}}(y) \mathrm{d} y+h_{0}(x)\right]\right\} \\
& -\bar{m}(x) \mid \\
\leqslant & \beta\left|\int_{\varepsilon^{-\frac{1}{2}}}^{x+1} J(x, y)\left[\hat{M}_{\mu_{0}}(y)-\bar{m}(y)\right] \mathrm{d} y+h_{0}(x)\right| \\
\leqslant & \beta\left|\hat{M}_{\mu_{0}}(x+1)-\bar{m}(x+1)\right|+O(\sqrt{\varepsilon}),
\end{aligned}
$$

because $\hat{M}_{\mu_{0}}$ is non increasing while $\bar{m}$ is increasing. Since by definition of $m_{0}$ $\hat{M}_{\mu_{0}}\left(\varepsilon^{-\frac{1}{2}}\right)=\bar{m}\left(\varepsilon^{-\frac{1}{2}}\right)$ :

$$
\begin{aligned}
\left|\hat{M}_{\mu_{0}}(x+1)-\bar{m}(x+1)\right| \leqslant & \left|\hat{M}_{\mu_{0}}(x+1)-\hat{M}_{\mu_{0}}\left(\varepsilon^{-\frac{1}{2}}\right)\right| \\
& +\left|\bar{m}(x+1)-\bar{m}\left(\varepsilon^{-\frac{1}{2}}\right)\right|=O(\varepsilon)
\end{aligned}
$$

because the first term at the right hand side is bounded by the sup norm of the derivative of $\hat{M}_{\mu_{0}}$, while the second term is exponentially small in $\varepsilon^{-\frac{1}{2}}$. Finally, if $\varepsilon^{-\frac{1}{2}} \leqslant x \leqslant \varepsilon^{-\frac{1}{2}}+1$ :

$$
\begin{aligned}
\left|S_{0}(x)\right|= & \mid \tanh \left\{\beta\left[\int_{x-1}^{\varepsilon^{-\frac{1}{2}}} J(x, y) \bar{m}(y) \mathrm{d} y+\int_{\varepsilon}^{-\frac{1}{2}}{ }^{x+1} J(x, y) \hat{M}_{\mu_{0}}(y) \mathrm{d} y+h_{0}(x)\right]\right\} \\
& -\hat{M}_{\mu_{0}}(x) \mid \\
\leqslant & \beta\left|\int_{x-1}^{\varepsilon^{-\frac{1}{2}}} J(x, y)\left[\bar{m}(y)-\hat{M}_{\mu_{0}}(y)\right] \mathrm{d} y+\int_{x-1}^{x+1} J(x, y) \hat{M}_{\mu_{0}}(y) \mathrm{d} y-\hat{M}_{\mu_{0}}(x)\right| \\
\leqslant & \beta\left|\bar{m}(x-1)-\hat{M}_{\mu_{0}}(x-1)\right|+\beta\left\|\hat{M}^{\prime}\right\|_{\varepsilon}=O(\varepsilon)
\end{aligned}
$$

by virtue of estimates (43) and (73). This proves that $\left\|S_{0}\right\|_{\varepsilon}=O(\varepsilon) . \mathrm{I}-\mathrm{A}_{m_{0}, h_{0}}$ is clearly invertible, then $\varphi_{1}$ exists and explicitly:

$$
\varphi_{1}(x)=\left(\mathrm{I}-\mathrm{A}_{m_{0}, h_{0}}\right)^{-1} S_{0}(x) .
$$

By Lemma 4.7, since $S_{0}(x)$ is antisymmetric, we also get the upper bound $\left\|\varphi_{1}\right\|_{\varepsilon} \leqslant$ $C\left(1-c_{\varepsilon} \gamma\right)^{-1}\left\|S_{0}\right\|_{\varepsilon} \leqslant c_{0} \varepsilon$ for some constant $c_{0}$.

Proposition 5.2 For any $\varepsilon$ small enough, we have 
(i) for any $k \geqslant 2$ there are antisymmetric functions $\varphi_{k} \in L^{\infty}\left(\left[-\varepsilon^{-1}, \varepsilon^{-1}\right]\right)$ that solve

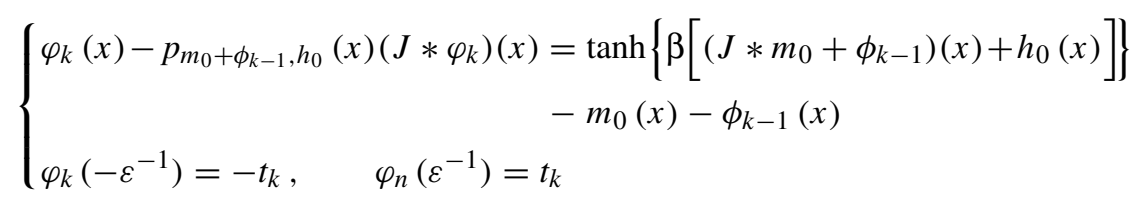

for some $t_{k} \in \mathbb{R}$, where $\phi_{k}$ is defined in (67) and $\varphi_{1}$ solves (84);

(ii) there is a constant $\tau$ such that $\left\|\varphi_{k}\right\|_{\varepsilon} \leqslant \tau\left\|\varphi_{k-1}\right\|_{\varepsilon}^{2}$ for any $k \geqslant 2$;

(iii) $\lim _{k \rightarrow \infty}\left\|m_{1}-m_{0}-\phi_{k}\right\|_{\varepsilon}=0$, where $m_{1}$ solves (34) with $n=1$;

(iv) $\left\|m_{1}-m_{0}\right\|_{\varepsilon}=O(\varepsilon)$.

Proof It works by induction. In particular, suppose that (i) and (ii) hold for any integer less or equal to a certain $k$. Under this hypothesis, since $\left\|\varphi_{1}\right\|_{\varepsilon} \leqslant c_{0} \varepsilon$, iterating (ii) we get

$$
\left\|\varphi_{k}\right\|_{\varepsilon} \leqslant \tau^{2^{k-1}-1}\left(c_{0} \varepsilon\right)^{2^{k-1}}
$$

and then, if $\varepsilon<\left(2 c_{0} \tau\right)^{-2}$ using the geometric series we get uniformly in $k$ :

$$
\left\|\phi_{k}\right\|_{\varepsilon} \leqslant 2 c_{0} \varepsilon \text {. }
$$

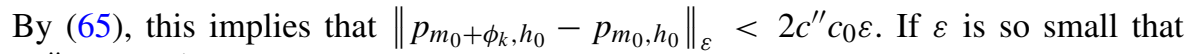
$2 c^{\prime \prime} c_{0} \varepsilon<\delta^{*}, \mathrm{I}-\mathrm{A}_{m_{0}+\phi_{k}, h_{0}}$ is invertible and $\varphi_{k+1}$ exists. We prove (ii); expand the hyperbolic tangent in Taylor series:

$$
\begin{aligned}
\tanh \left\{\beta\left[\left(J * m_{0}+\phi_{k}\right)(x)+h_{0}(x)\right]\right\}= & \tanh \left\{\beta\left[\left(J * m_{0}+\phi_{k-1}\right)(x)+h_{0}(x)\right]\right\} \\
& +p_{m_{0}+\phi_{k-1}, h_{0}}(x)\left(J * \varphi_{k}\right)(x) \\
& +p_{m_{0}+\phi_{k-1}, h_{0}}^{\prime}(x)\left(J * \varphi_{k}\right)^{2}(x)+\ldots
\end{aligned}
$$

Combining (78) with the definition of $\varphi_{k}$ we get

$$
\varphi_{k+1}(x)=\left(\mathrm{I}-\mathrm{A}_{m_{0}+\phi_{k}, h_{0}}\right)^{-1}\left(p_{m_{0}+\phi_{k-1}, h_{0}}^{\prime}(x)\left(J * \varphi_{k}\right)(x)+\ldots\right),
$$

hence

$$
\left\|\varphi_{k+1}\right\|_{\varepsilon} \leqslant \frac{C}{1-c_{\varepsilon} \gamma} \sup _{\left|x^{\prime}\right| \leq \varepsilon^{-1}} p_{m_{0}+\phi_{k-1}, h_{0}}^{\prime}\left(x^{\prime}\right) \sup _{\left|x^{\prime \prime}\right| \leq \varepsilon^{-1}} \varphi_{k}^{2}\left(x^{\prime \prime}\right) \leqslant \frac{\beta C}{1-c_{\varepsilon} \gamma}\left\|\varphi_{k}\right\|_{\varepsilon}^{2}
$$

so we can identify $\tau=\beta C /\left(1-c_{\varepsilon} \gamma\right)$. This proves that for any integer $k$ :

$$
m_{0}(x)+\phi_{k}(x)=\tanh \left\{\beta\left[\left(J * m_{0}+\phi_{k}\right)(x)+h_{0}(x)\right]\right\}+O\left(\varphi_{k}(x)\right) .
$$

Taking the limit $k \rightarrow \infty$, by continuity of the hyperbolic tangent we get the uniform convergence to $m_{1}$. (iv) follows from (77). 


\subsection{Small Perturbations to $h_{0}$}

Proposition 5.3 There is $\delta^{\prime}>0$ such that for any $h \in C\left(\left[-\varepsilon^{-1}, \varepsilon^{-1}\right]\right)$, if $\left\|h-h_{0}\right\|_{\varepsilon} \leqslant \delta^{\prime}$ :

(i) there exists a continuous, antisymmetric function $m$ which is a solution of

$$
m(x)=\tanh \{\beta[(J * m)(x)+h(x)]\} ;
$$

(ii) $\left\|m-m_{0}\right\|_{\varepsilon}=O\left(\delta^{\prime}\right)$.

Proof We construct $m$ using again Newton's method with starting point $\left(m_{0}, h\right)$. Observe that

$$
\left\|p_{m_{0}, h}-p_{m_{0}, h_{0}}\right\|_{\varepsilon} \leqslant 4 \beta \tanh \left\{\beta\left\|h-h_{0}\right\|_{\varepsilon}\right\} \leqslant 4 \beta^{2} \delta^{\prime}
$$

hence, if $\delta^{\prime}<\delta^{*} / 4 \beta^{2}$, we are in the hypothesis of Lemma 4.7 , that combined with Proposition 5.2 gives the existence of an antisymmetric function $\psi_{1}$ that solves

$$
\left\{\begin{array}{l}
\psi_{1}(x)-p_{m_{0}, h}(x)\left(J * \psi_{1}\right)(x)=\tanh \left\{\beta\left[\left(J * m_{0}\right)(x)+h(x)\right]\right\}-m_{0}(x) \\
\psi_{1}\left(-\varepsilon^{-1}\right)=-s_{1}, \quad \psi_{1}\left(\varepsilon^{-1}\right)=s_{1}
\end{array}\right.
$$

for some $s_{1} \in \mathbb{R}$, and moreover $\left\|\psi_{1}\right\|_{\varepsilon} \leqslant \tau\left\|h-h_{0}\right\|_{\varepsilon}$. The rest of the proof is the same as that of Proposition 5.2, provided $\delta^{\prime}$ is so small that Lemma 4.7 can be applied recursively.

\subsection{Further corrections}

In order to emphasize scaling properties of the magnetization profile, we introducing the following weighted norm at fixed $\alpha>0$ :

$$
\|m\|_{\varepsilon, \alpha}:=\sup _{|x| \leq \varepsilon^{-1}} \mathrm{e}^{-\alpha \varepsilon|x|}|m(x)|, \quad m \in L^{\infty}\left(\left[-\varepsilon^{-1}, \varepsilon^{-1}\right]\right) .
$$

Notice that convergence in the $\alpha$-norm implies uniform convergence as the inclusion $\|\cdot\|_{\varepsilon, \alpha} \leqslant\|\cdot\|_{\varepsilon} \leqslant \mathrm{e}^{\alpha}\|\cdot\|_{\varepsilon, \alpha}$ holds. The iterability of our method directly follows from the fact that $\left(m_{n}, h_{n}\right) \mapsto\left(m_{n+1}, h_{n+1}\right)$ is a contraction in this norm (for a feasible choice of parameters).

Lemma 5.4 Let $^{(i)}, i=1,2$ such that $\left\|h^{(i)}-h_{0}\right\|_{\varepsilon} \leq \delta^{\prime}$, and $m^{(i)}$ the corresponding profiles constructed via Newton's method that solve

$$
m^{(i)}(x)=\tanh \left\{\beta\left[\left(J * m^{(i)}\right)(x)+h^{(i)}(x)\right]\right\} .
$$

Then,

$$
\left\|m^{(2)}-m^{(1)}\right\|_{\varepsilon, \alpha} \leqslant \tau\left\|h^{(2)}-h^{(1)}\right\|_{\varepsilon, \alpha}
$$

for $\varepsilon$ small enough. 
Proof $m^{(1)}$ and $m^{(2)}$ exist by Proposition 5.3. By Taylor's Theorem

$$
m^{(2)}(x)=\tanh \left\{\beta\left[\left(J * m^{(1)}\right)(x)+h^{(1)}(x)\right]\right\}+R_{1,2}(x),
$$

where

$$
R_{1,2}(x)=p_{1,2}(x)\left[\left(J *\left(m^{(2)}-m^{(1)}\right)\right)(x)+h^{(2)}(x)-h^{(1)}(x)\right]
$$

$p_{1,2}$ being some interpolating function between $p_{m^{(2)}, h^{(2)}}$ and $p_{m^{(1)}, h^{(1)}}$. We multiply by $\mathrm{e}^{-\alpha \varepsilon|x|}(88)$ and take absolute values to get

$$
\begin{aligned}
& \mathrm{e}^{-\alpha \varepsilon|x|}\left|m^{(2)}(x)-m^{(1)}(x)\right|-p_{1,2}(x) \mathrm{e}^{-\alpha \varepsilon}\left(J *\left|m^{(2)}-m^{(1)}\right|\right)(x) \\
\leqslant & p_{1,2}(x) \mathrm{e}^{-\alpha \varepsilon|x|}\left|h^{(2)}(x)-h^{(1)}(x)\right| .
\end{aligned}
$$

(90) as an equality can be solved in the unknown function $\mathrm{e}^{-\alpha \varepsilon|x|}\left|m^{(2)}(x)-m^{(1)}(x)\right|$ provided $\left\|p_{1,2} \mathrm{e}^{\alpha \varepsilon}-p_{m_{0}, h_{0}}\right\|_{\varepsilon}<\delta^{*}$. Then, for $\varepsilon$ small enough:

$$
\left\|m^{(2)}-m^{(1)}\right\|_{\varepsilon, \alpha} \leqslant \frac{C\left\|p_{1,2}\right\|_{\varepsilon}}{1-c_{\varepsilon} \gamma}\left\|h^{(2)}-h^{(1)}\right\|_{\varepsilon, \alpha} \leqslant \tau\left\|h^{(2)}-h^{(1)}\right\|_{\varepsilon, \alpha} .
$$

\subsection{Convergence to $(m, h)$}

We now prove Proposition 3.3.

Proof Suppose that for any $k<n, n$ a fixed integer, the following hypothesis hold true:

(H1) there is a continuous, antisymmetric function $m_{k}$ which solves

$$
m_{k}(x)=\tanh \left\{\beta\left[\left(J * m_{k}\right)(x)+h_{k-1}(x)\right]\right\}
$$

where $h_{k}=\mathrm{H}_{j}\left(m_{k}\right)$, and boundary conditions $m_{k}\left(-\varepsilon^{-1}\right)=-\mu_{k}$, $m_{k}\left(\varepsilon^{-1}\right)=\mu_{k}, \mu_{k} \in\left(m^{*}(\beta), m_{\beta}\right)$.

(H2) there is a constant $\rho \in(0,1)$ independent of $k$ such that

$$
\left\|h_{k}-h_{k-1}\right\|_{\varepsilon, \alpha} \leqslant \rho\left\|h_{k-1}-h_{k-2}\right\|_{\varepsilon, \alpha}, \quad 2 \leqslant k<n .
$$

Notice that (H1) and (H2) imply:

$$
\begin{gathered}
\left\|h_{k}-h_{k-1}\right\|_{\varepsilon, \alpha} \leqslant \frac{\rho}{1-\rho}\left\|h_{1}-h_{0}\right\|_{\varepsilon, \alpha} \\
\left\|h_{k}-h_{0}\right\|_{\varepsilon, \alpha} \leqslant \frac{\rho}{1-\rho}\left\|h_{1}-h_{0}\right\|_{\varepsilon, \alpha} .
\end{gathered}
$$

Moreover, (95) reads in the sup norm

$$
\left\|h_{k}-h_{0}\right\|_{\varepsilon} \leqslant \frac{\mathrm{e}^{\alpha} \rho}{1-\rho}\left\|h_{1}-h_{0}\right\|_{\varepsilon} .
$$


We know that $\left\|h_{1}-h_{0}\right\|_{\varepsilon}=O(\varepsilon)$; at fixed $\alpha$, we choose $\varepsilon$ so small that $\left\|h_{k}-h_{0}\right\|_{\varepsilon} \leqslant \delta^{*}$ uniformly in $k$. This way Lemma 5.4 applies and then there exists $m_{n}$ solution of

$$
m_{n}(x)=\tanh \left\{\beta\left[\left(J * m_{n}\right)(x)+h_{n-1}(x)\right]\right\}
$$

satisfying certain boundary conditions. It remains to prove that (H2) holds for $k=n$. We have, for any $x \in\left[-\varepsilon^{-1}, \varepsilon^{-1}\right]$ :

$$
\begin{aligned}
\left|h_{n}(x)-h_{n-1}(x)\right| & \leqslant c^{\prime} j \varepsilon \int_{0}^{x} \mathrm{e}^{-\alpha \varepsilon y}\left|m_{n}(x)-m_{n-1}(x)\right| \mathrm{e}^{\alpha \varepsilon y} \mathrm{~d} y \\
& \leqslant c^{\prime} j \varepsilon\left\|m_{n}-m_{n-1}\right\|_{\varepsilon, \alpha} \int_{0}^{x} \mathrm{e}^{\alpha \varepsilon y} \mathrm{~d} y \\
& \leqslant \frac{c^{\prime} j}{\alpha}\left\|m_{n}-m_{n-1}\right\|_{\varepsilon, \alpha} .
\end{aligned}
$$

Hence, in the $\alpha$-norm:

$$
\left\|h_{n}-h_{n-1}\right\|_{\varepsilon, \alpha} \leqslant \frac{c^{\prime} j}{\alpha}\left\|m_{n}-m_{n-1}\right\|_{\varepsilon, \alpha} \leqslant \frac{c^{\prime} j_{\max } \tau}{\alpha}\left\|h_{n-1}-h_{n-2}\right\|_{\varepsilon, \alpha},
$$

where the second inequality follows from (87). Let then $\alpha>c^{\prime} j_{\max } \tau$ and choose $\rho=c^{\prime} j_{\max } \tau / \alpha$. If $\varepsilon$ is so small that (96) is bounded by $\delta^{*},\left(m_{n}, h_{n}\right) \mapsto\left(m_{n+1}, h_{n+1}\right)$ is a contraction in the $\alpha$-norm. This implies uniform convergence to a solution of (27).

\section{Invertibility of the Method}

We proved so far that at fixed $\beta>1$, one can choose any $\mu_{0} \in\left(m^{*}(\beta), m_{\beta}\right)$ and get a solution $m$ of problem (23) satisfying a certain boundary condition $m\left(\varepsilon^{-1}\right)=\mu$ for any $\varepsilon$ small enough. $\varepsilon$ actually depends on $\mu_{0}$ as most of the constants appearing in the bounds obtained in the previous sections do. It is not possible to get a uniform bound (in $\varepsilon$ ) on the open domain $\mu_{0} \in\left(m^{*}(\beta), m_{\beta}\right)$ for two reasons. The first one is that some quantities diverge as $\mu_{0} \rightarrow m^{*}(\beta)$; the second one is that we are interested in solutions carrying positive current while, according to (35), at fixed $\varepsilon$ there are infinite values of $\mu_{0}$ such that $j<0$. Nonetheless, we can cover any subset of the metastable domain taking $\varepsilon$ feasibly small.

We indicate in this section $m_{0}(\cdot, j)$ as the starting magnetization profile carrying current $j$. This also fix the boundary value $m_{0}\left(\varepsilon^{-1}\right)=\mu_{0}$. We use the same notation for the corresponding auxiliary magnetic field.

\subsection{Lipschitz Continuity in $j$}

Proposition 6.1 The sequence $\left(m_{n}(\cdot, j)\right)_{n=0}^{\infty}$ constructed via Newton's method is Lipschitz continuous in $j$ in any subset of $\left(j\left(m_{\beta}\right), j\left(m^{*}(\beta)+\zeta\right)\right), \zeta>0$ arbitrary chosen. The limit profile $m(\cdot, j)$ is Lipschitz continuous in $j$ as well. 
Proof We differentiate $m_{0}$ and $h_{0}$ with respect to $j$ :

$$
\begin{aligned}
& \frac{\partial m_{0}}{\partial j}(x, j)= \begin{cases}0 & 0 \leqslant x \leqslant \varepsilon^{-\frac{1}{2}} \\
-\frac{1}{1-\chi_{\beta}\left(m_{0}(x, j)\right)} \frac{\varepsilon x-\sqrt{\varepsilon}}{1-\sqrt{\varepsilon}} & \varepsilon^{-\frac{1}{2}} \leqslant x \leqslant \varepsilon^{-1},\end{cases} \\
& \frac{\partial h_{0}}{\partial j}(x, j)= \begin{cases}0 & 0 \leqslant x \leqslant \varepsilon^{-\frac{1}{2}} \\
-\frac{1}{\chi_{\beta}\left(m_{0}(x, j)\right)} \frac{\varepsilon x-\sqrt{\varepsilon}}{1-\sqrt{\varepsilon}} & \varepsilon^{-\frac{1}{2}} \leqslant x \leqslant \varepsilon^{-1}\end{cases}
\end{aligned}
$$

and similarly for $x<0$. Then, for any $j_{1}$ and $j_{2}$, using Lagrange's Theorem:

$$
\begin{aligned}
& \left\|m_{0}\left(\cdot, j_{2}\right)-m_{0}\left(\cdot, j_{1}\right)\right\|_{\varepsilon} \leqslant\left(1-\chi_{\beta}\left(m^{*}(\beta)+\zeta\right)\right)^{-1}\left|j_{2}-j_{1}\right|, \\
& \left\|h_{0}\left(\cdot, j_{2}\right)-h_{0}\left(\cdot, j_{1}\right)\right\|_{\varepsilon} \leqslant \chi_{\beta}^{-1}\left(m_{\beta}\right)\left|j_{2}-j_{1}\right| .
\end{aligned}
$$

Performing similar computations to those of proof of Lemma 5.4, it can be shown that for any $n>0$

$$
\left\|m_{n+1}\left(\cdot, j_{2}\right)-m_{n+1}\left(\cdot, j_{1}\right)\right\|_{\varepsilon, \alpha} \leqslant \tau\left\|h_{n}\left(\cdot, j_{2}\right)-h_{n}\left(\cdot, j_{1}\right)\right\|_{\varepsilon, \alpha} .
$$

At fixed $n$, compute then

$$
\begin{aligned}
\left|h_{n}\left(x, j_{2}\right)-h_{n}\left(x, j_{1}\right)\right| & \leqslant \varepsilon\left|\int_{0}^{x} \frac{j_{2} \mathrm{~d} y}{\chi_{\beta}\left(m_{n}\left(y, j_{2}\right)\right)}-\int_{0}^{x} \frac{j_{1} \mathrm{~d} y}{\chi_{\beta}\left(m_{n}\left(y, j_{1}\right)\right)}\right| \\
& \leqslant \frac{c^{\prime}}{2} \varepsilon \int_{0}^{x}\left(\left|j_{2}-j_{1}\right|+\left|j_{2} m_{n}^{2}\left(y, j_{1}\right)-j_{1} m_{n}^{2}\left(y, j_{2}\right)\right|\right) \mathrm{d} y .
\end{aligned}
$$

Adding and subtracting the term $j_{2} m_{n}^{2}\left(y, j_{2}\right)$ we get

$$
\left|h_{n}\left(x, j_{2}\right)-h_{n}\left(x, j_{1}\right)\right| \leqslant c^{\prime} \varepsilon\left(\left|j_{2}-j_{1}\right| \varepsilon^{-1}+j_{2} \int_{0}^{x}\left|m_{n}\left(y, j_{2}\right)-m_{n}\left(y, j_{1}\right)\right| \mathrm{d} y\right)
$$

that in the $\alpha$-norm reads

$$
\begin{aligned}
\left\|h_{n}\left(\cdot, j_{2}\right)-h_{n}\left(\cdot, j_{1}\right)\right\|_{\varepsilon, \alpha} & \leqslant c^{\prime}\left|j_{2}-j_{1}\right|+\frac{c^{\prime} j_{2}}{\alpha}\left\|m_{n}\left(\cdot, j_{2}\right)-m_{n}\left(\cdot, j_{1}\right)\right\|_{\varepsilon, \alpha} \\
& \leqslant c^{\prime}\left|j_{2}-j_{1}\right|+\frac{c^{\prime} \tau j_{2}}{\alpha}\left\|h_{n-1}\left(\cdot, j_{2}\right)-h_{n-1}\left(\cdot, j_{1}\right)\right\|_{\varepsilon, \alpha}
\end{aligned}
$$

where we used (104). By iteration, we get

$$
\begin{aligned}
\left\|h_{n}\left(\cdot, j_{2}\right)-h_{n}\left(\cdot, j_{1}\right)\right\|_{\varepsilon, \alpha} \leqslant & c^{\prime}\left[\sum_{k=0}^{n-1}\left(\frac{c^{\prime} \tau j_{2}}{\alpha}\right)^{k}\right]\left|j_{2}-j_{1}\right| \\
& +c^{\prime}\left(\frac{c^{\prime} \tau j_{2}}{\alpha}\right)^{n}\left\|h_{0}\left(\cdot, j_{2}\right)-h_{0}\left(\cdot, j_{1}\right)\right\|_{\varepsilon, \alpha} .
\end{aligned}
$$


Then, recalling (103) we obtain the uniform bound

$$
\left\|h_{n}\left(\cdot, j_{2}\right)-h_{n}\left(\cdot, j_{1}\right)\right\|_{\varepsilon, \alpha} \leqslant c^{\prime \prime \prime}\left|j_{2}-j_{1}\right|,
$$

$c^{\prime \prime \prime}$ a constant. Given (104), we finally get in the sup norm

$$
\left\|m_{n}\left(\cdot, j_{2}\right)-m_{n}\left(\cdot, j_{1}\right)\right\|_{\varepsilon} \leqslant \tau c^{\prime \prime \prime} \mathrm{e}^{\alpha}\left|j_{2}-j_{1}\right| .
$$

The fact that the function $m$ that is obtained as limit of Newton's method is Lipschitz is a consequence of the uniform continuity of the sequence.

\subsection{Proof of Proposition 3.4}

This closes the proof of Theorem 3.1.

Proof Suppose that we want to solve problem (23) with boundary condition $m\left(\varepsilon^{-1}\right)=\mu$, where $\mu \in\left(m^{*}(\beta), m_{\beta}\right)$. Let $\Delta>0$ a constant such that $(\mu-\Delta, \mu+\Delta) \subset\left(m^{*}(\beta), m_{\beta}\right)$. For any $\mu_{0} \in(\mu-\Delta, \mu+\Delta)$, we proved that there is a constant $c_{\Delta}$ such that $\left\|m\left(\cdot, j\left(\mu_{0}\right)\right)-m_{0}\left(\cdot, j\left(\mu_{0}\right)\right)\right\|_{\varepsilon} \leqslant c_{\Delta} \varepsilon$. Let $\varepsilon$ so small that $\varepsilon<\frac{\Delta}{2 c_{\Delta}}$. Define $\mu_{0}^{(-)}:=\mu-c_{\Delta} \varepsilon$ and $\mu_{0}^{(+)}:=\mu+c_{\Delta} \varepsilon$, and accordingly $j^{(-)}:=j\left(\mu_{0}^{(-)}\right), j^{(+)}:=j\left(\mu_{0}^{(+)}\right)$. By hypothesis, $m\left(\varepsilon^{-1}, j^{(-)}\right)>\mu$ and $m\left(\varepsilon^{-1}, j^{(+)}\right)<\mu$ (recall that $j$ in non increasing as a function of the boundary condition). By Proposition 6.1, $m$ is Lipschitz in $j$ in $(\mu-\Delta, \mu+\Delta)$, then continuous. We then apply the intermediate value theorem to conclude that $m\left(\varepsilon^{-1}, j\right)$ takes all the values between $m\left(\varepsilon^{-1}, j^{(+)}\right)$and $m\left(\varepsilon^{-1}, j^{(-)}\right)$, then in particular $\mu$.

Acknowledgements We thank Anna De Masi, Errico Presutti and Dimitrios Tsagkarogiannis for enlightening discussions.

Funding Information Open access funding provided by Università degli Studi dell' Aquila within the CRUI-CARE Agreement.

Open Access This article is licensed under a Creative Commons Attribution 4.0 International License, which permits use, sharing, adaptation, distribution and reproduction in any medium or format, as long as you give appropriate credit to the original author(s) and the source, provide a link to the Creative Commons licence, and indicate if changes were made. The images or other third party material in this article are included in the article's Creative Commons licence, unless indicated otherwise in a credit line to the material. If material is not included in the article's Creative Commons licence and your intended use is not permitted by statutory regulation or exceeds the permitted use, you will need to obtain permission directly from the copyright holder. To view a copy of this licence, visit http://creativecommonshorg/licenses/by/4.0/.

\section{References}

1. Giacomin, G.B., Lebowitz, J.L.: Phase segregation dynamics in particle system with long range interactions. J. Stat. Phys. 87(1), 37-61 (1997)

2. Bertini, L., De Sole, A., Gabrielli, D., Jona-Lasinio, G., Landim, C.: Fluctuations in stationary nonequilibrium states of irreversible processes. Phys. Rev. Lett. 87(4):040601 (2001)

3. Bertini, L., De Sole, A., Gabrielli, D., Jona-Lasinio, G., Landim, C.: Macroscopic fluctuation theory for stationary non-equilibrium states. J. Stat. Phys. 107(3-4):635-675 
4. Bertini, L., De Sole, A., Gabrielli, D., Jona-Lasinio, G., Landim, C.: Current fluctuations in stochastic lattice gases. Phys. Rev. Lett. 94(3):030601

5. Nernst, W.: Z. physik. Chem. 2, 613 (1888)

6. Onsager, L.: Theories and problems of liquid diffusion. Ann. N. Y. Acad. Sci. 46(5), 241-265 (1945)

7. Darken, L.S., Larsen, B.M.: Distribution of manganese and of sulphur between slag and metal in the open-hearth furnace. Trans. Aime 150, 87-112 (1942)

8. Darken, L.S.: Diffusion, mobility and their interrelation through free energy in binary metallic systems. Trans. Aime 175, 184-201 (1948)

9. Darken, L.S.: Diffusion of carbon in austenite with a discontinuity in composition. Trans. Aime 180(53), 430-438 (1949)

10. Krishna, R.: Uphill diffusion in multicomponent mixtures. Chem. Soc. Rev. 44(10), 2812-2836 (2015)

11. Krishna, R.: Serpentine diffusion trajectories and the Ouzo effect in partially miscible ternary liquid mixtures. Phys. Chem. Chem. Phys. 17(41), 27428-27436 (2015)

12. Colangeli, M., De Masi, A., Presutti, E.: Particle models with self sustained current. J. Stat. Phys. 167(5), 1081-1111 (2017)

13. Boccagna, R.: Fick's law and phase transitions. Ph.D. Thesis (unpublished) (2019)

14. Colangeli, M., Giardinà, C., Giberti, C., Vernia, C.: Nonequilibrium two-dimensional Ising model with stationary uphill diffusion. Phys. Rev. E 97(3):030103 (2018)

15. De Masi, A., Orlandi, E., Presutti, E., Triolo, L.: Uniqueness and global stability of the instanton in nonlocal evolution equations. Rend. Mat. Delle Appl. 14, 693-723 (1994)

16. De Masi, A., Orlandi, E., Presutti, E., Triolo, L.: Stability of the interface in a model of phase separation. Proc. R. Soc. Edinb. Sect. Math. 124(5), 1013-1022 (1994)

17. De Masi, A., Orlandi, E., Presutti, E., Triolo, L.: Motion by curvature by scaling non local evolution equations. J. Phys. 73, 543-570 (1993)

18. De Masi, A., Orlandi, E., Presutti, E., Triolo, L.: Glauber evolution with Kac potentials. I. Mesoscopic and macroscopic limits, interface dynamics. Nonlinearity 7(3), 633 (1994)

19. De Masi, A., Presutti, E., Tsagkarogiannis, D.: Fourier law, phase transitions and the stationary Stefan problem. Arch. Ration. Mech. Anal. 201(2), 681-725 (2011)

20. Presutti, E.: Scaling limits in statistical mechanics and microstructures in continuum mechanics. Springer Science \& Business Media (2008)

21. Boccagna, R.: Fick's law in non-local evolution equations. J. Math. Phys. 59(5):053508 (2018)

22. Boccagna, R.: Current with "wrong" sign and phase transitions. J. Math. Phys. 60(4):043509 (2019)

23. De Masi, A., Olivieri, E., Presutti, E.: Critical droplet for a non local mean field equation. Markov Process. Related Fields 6, 439-472 (2000)

24. De Masi, a., Olivieri, E., Presutti, E.: Spectral properties of integral operators in problems of interface dynamics and metastability. Markov Process. Related Fields 4.1, 27-112 (1998)

Publisher's Note Springer Nature remains neutral with regard to jurisdictional claims in published maps and institutional affiliations. 